\title{
A Note on Multiple-Access Channels with Strictly-Causal State Information
}

\author{
Amos Lapidoth \\ ETH Zurich \\ Switzerland \\ Email: lapidoth@isi.ee.ethz.ch
}

\author{
Yossef Steinberg \\ Technion-Israel Institute of Technology \\ Israel \\ Email: ysteinbe@ee.technion.ac.il
}

\begin{abstract}
We propose a new inner bound on the capacity region of a memoryless multiple-access channel that is governed by a memoryless state that is known strictly causally to the encoders. The new inner bound contains the previous bounds, and we provide an example demonstrating that the inclusion can be strict.

A variation on this example is then applied to the case where the channel is governed by two independent state sequences, where each transmitter knows one of the states strictly causally. The example proves that, as conjectured by $\mathrm{Li}$ et al., an inner bound that they derived for this scenario can indeed by strictly better than previous bounds.
\end{abstract}

\section{INTRODUCTION}

If a memoryless single-user channel is governed by an independent and identically distributed (IID) state sequence, then its capacity is not increased if the state is made available to the encoder in a strictly-causal way. The picture changes dramatically on the multiple-access channel (MAC) [1], [2]: In the "single-state scenario," where the channel is governed by a single state sequence, the capacity region typically increases if the state is revealed to both transmitters in a strictly causal way [1]. Some of the gains can be attributed to the ability of the two encoders to compress the state information and to cooperate in sending the compressed version to the receiver. But strictly-causal side information (SI) is beneficial even in the "double-state scenario," where the channel is governed by two independent states, with each transmitter knowing one of the sequences strictly causally. In this case too, the side information can be helpful even though the transmitters cannot cooperate in compressing the states or in sending them [2].

The present note deals with both the single-state and the double-state scenarios. For the single-state scenario, we present a new inner bound on the capacity region. This bound contains the inner bound of [1] (which was extended to the many-transmitters scenario in [3]). We also provide an example showing that the inclusion can be strict.

By adapting this example to the double-state scenario, we provide an example showing that-as conjectured in [3] - the inner bound proposed by Li et al. in [3] can be strictly larger than that in [2].

To keep the contribution focused, we do not consider causal side information in this note, although our results can be carried over to that setting as in [1], [2].
We next describe the two scenarios more explicitly. Our descriptions are identical to those in [1], [2] except that, for simplicity, we do not consider cost constraints and we assume throughout that all the alphabets are finite.

\section{A. The Single-State Scenario}

In the single-state scenario we are given a discrete memoryless state-dependent MAC of law $P_{Y \mid W, X_{1}, X_{2}}$ with state alphabet $\mathcal{W}$, state probability mass function (PMF) $P_{W}$, input alphabets $\mathcal{X}_{1}$ and $\mathcal{X}_{2}$, and output alphabet $\mathcal{Y}$. Sequences of letters from $\mathcal{W}$ are denoted $w^{n}=\left(w_{1}, w_{2}, \ldots, w_{n}\right)$ and $w_{i}^{j}=\left(w i, w_{i+1} \ldots, w_{j}\right)$. Similar notation holds for all alphabets, e.g. $x_{1}^{n}=\left(x_{1,1}, x_{1,2}, \ldots, x_{1, n}\right), x_{2, i}^{j}=$ $\left(x_{2, i}, x_{2, i+1}, \ldots, x_{2, j}\right)$. When there is no risk of ambiguity, $n$-sequences will sometimes be denoted by boldface letters, $\mathbf{y}$, $\mathbf{x}_{1}$, w, etc. The laws governing $n$-sequences of output letters and states are

$$
\begin{gathered}
P_{Y \mid W, X_{1}, X_{2}}^{n}\left(\mathbf{y} \mid \mathbf{w}, \mathbf{x}_{1}, \mathbf{x}_{2}\right)=\prod_{i=1}^{n} P_{Y \mid W, X_{1}, X_{2}}\left(y_{i} \mid w_{i}, x_{1, i}, x_{2, i}\right), \\
P_{W}^{n}(\mathbf{w})=\prod_{i=1}^{n} P_{W}\left(w_{i}\right) .
\end{gathered}
$$

For notational convenience, we henceforth omit the superscript $n$, and we denote the channel by $P$.

Definition 1: Given positive integers $\nu_{1}, \nu_{2}$, let $\mathcal{M}_{1}$ denote the set $\left\{1,2, \ldots, \nu_{1}\right\}$, and let $\mathcal{M}_{2}$ denote the set $\left\{1,2, \ldots, \nu_{2}\right\}$. An $\left(n, \nu_{1}, \nu_{2}, \epsilon\right)$ code with strictly-causal side information (SI) at the encoders is a pair of sequences of encoder mappings

$$
f_{k, i}: \mathcal{W}^{i-1} \times \mathcal{M}_{k} \rightarrow \mathcal{X}_{k}, \quad k=1,2, \quad i=1, \ldots, n
$$

and a decoding mapping

$$
g: \mathcal{Y}^{n} \rightarrow \mathcal{M}_{1} \times \mathcal{M}_{2}
$$

such that the average probability of error $P_{\mathrm{e}}$ does now exceed $\epsilon$. Here $P_{\mathrm{e}}$ is $1-P_{\mathrm{c}}$;

$$
P_{\mathrm{c}}=\frac{1}{\nu_{1} \nu_{2}} \sum_{m_{1}=1}^{\nu_{1}} \sum_{m_{2}=1}^{\nu_{2}} \operatorname{Pr}\left(\text { correct } \mid m_{1}, m_{2}\right) ;
$$


and

$$
\begin{aligned}
& \operatorname{Pr}\left(\text { correct } \mid m_{1}, m_{2}\right)= \\
& \sum_{\mathbf{w}} P_{W}(\mathbf{w}) P\left(g^{-1}\left(m_{1}, m_{2}\right) \mid \mathbf{w}, \mathbf{f}_{1}\left(\mathbf{w}, m_{1}\right), \mathbf{f}_{2}\left(\mathbf{w}, m_{2}\right)\right),
\end{aligned}
$$

where $g^{-1}\left(m_{1}, m_{2}\right) \subset \mathcal{Y}^{n}$ is the decoding set of the pair of messages $\left(m_{1}, m_{2}\right)$, and

$\mathbf{f}_{k}\left(\mathbf{w}, m_{k}\right)=\left(f_{k, 1}\left(m_{k}\right), f_{k, 2}\left(w_{1}, m_{k}\right), \ldots, f_{k, n}\left(w^{n-1}, m_{k}\right)\right)$.

The rate pair $\left(R_{1}, R_{2}\right)$ of the code is defined as

$$
R_{1}=\frac{1}{n} \log \nu_{1}, \quad R_{2}=\frac{1}{n} \log \nu_{2} .
$$

A rate-pair $\left(R_{1}, R_{2}\right)$ is said to be achievable if for every positive $\epsilon$ and sufficiently large $n$ there exists an $\left(n, 2^{n R_{1}}, 2^{n R_{2}}, \epsilon\right)$ code with strictly-causal SI for the channel $P_{Y \mid W, X_{1}, X_{2}}$. The capacity region of the channel with strictly-causal SI is the closure of the set of all achievable pairs $\left(R_{1}, R_{2}\right)$, and is denoted $\mathcal{C}_{\mathrm{s}-\mathrm{c}}^{\mathrm{com}}$. The subscript "s-c" stands for strictly-causal.

\section{B. The Double-State Scenario}

In the double-state scenario we are given a discrete memoryless state-dependent MAC $P_{Y \mid S_{1}, S_{2}, X_{1}, X_{2}}$ with state alphabets $\mathcal{S}_{1}$ and $\mathcal{S}_{2}$, state probability mass functions (PMFs) $P_{S_{1}}$ and $P_{S_{2}}$, input alphabets $\mathcal{X}_{1}$ and $\mathcal{X}_{2}$, and output alphabet $\mathcal{Y}$. The laws governing $n$ sequences of output letters and states are

$$
\begin{gathered}
P_{Y \mid S_{1}, S_{2}, X_{1}, X_{2}}^{n}\left(\mathbf{y} \mid \mathbf{s}_{1} \mathbf{s}_{2}, \mathbf{x}_{1}, \mathbf{x}_{2}\right) \\
=\prod_{i=1}^{n} P_{Y \mid S_{1}, S_{2}, X_{1}, X_{2}}\left(y_{i} \mid s_{1, i}, s_{2, i}, x_{1, i}, x_{2, i}\right), \\
P_{S_{1}, S_{2}}^{n}\left(\mathbf{s}_{1}, \mathbf{s}_{2}\right)=\prod_{i=1}^{n} P_{S_{1}}\left(s_{1, i}\right) P_{S_{2}}\left(s_{2, i}\right) .
\end{gathered}
$$

For notational convenience, we henceforth omit the superscript $n$, and we denote the channel by $P$.

Given positive integers $\nu_{1}, \nu_{2}$, let $\mathcal{M}_{1}$ be the set $\left\{1,2, \ldots, \nu_{1}\right\}$ and $\mathcal{M}_{2}$ the set $\left\{1,2, \ldots, \nu_{2}\right\}$. An $\left(n, \nu_{1}, \nu_{2}, \epsilon\right)$ code with strictly causal independent SI at the encoders is a pair of sequences of encoder mappings

$$
f_{k, i}: \mathcal{S}_{k}^{i-1} \times \mathcal{M}_{k} \rightarrow \mathcal{X}_{k}, \quad k=1,2, \quad i=1, \ldots, n
$$

and a decoding mapping

$$
g: \mathcal{Y}^{n} \rightarrow \mathcal{M}_{1} \times \mathcal{M}_{2}
$$

such that the average probability of error $P_{e}$ is bounded by $\epsilon$, where $P_{\mathrm{e}}=1-P_{\mathrm{c}}$ and

$$
\begin{gathered}
P_{\mathrm{c}}=\frac{1}{\nu_{1} \nu_{2}} \sum_{m_{1}=1}^{\nu_{1}} \sum_{m_{2}=1}^{\nu_{2}} \sum_{\mathbf{s}_{1}, \mathbf{s}_{2}} \\
P_{S_{1}}\left(\mathbf{s}_{1}\right) P_{S_{2}}\left(\mathbf{s}_{2}\right) P\left(g^{-1}\left(m_{1}, m_{2}\right) \mid \mathbf{s}_{1}, \mathbf{s}_{2}, \mathbf{f}_{1}\left(\mathbf{s}_{1}, m_{1}\right), \mathbf{f}_{2}\left(\mathbf{s}_{2}, m_{2}\right)\right)
\end{gathered}
$$

where $g^{-1}\left(m_{1}, m_{2}\right) \subset \mathcal{Y}^{n}$ is the decoding set of the pair of messages $\left(m_{1}, m_{2}\right)$, and

$\mathbf{f}_{k}\left(\mathbf{s}_{k}, m_{k}\right)=\left(f_{k, 1}\left(m_{k}\right), f_{k, 2}\left(s_{k, 1}, m_{k}\right), \ldots, f_{k, n}\left(s_{k}^{n-1}, m_{k}\right)\right)$.
The rate pair $\left(R_{1}, R_{2}\right)$ of the code is defined as

$$
R_{1}=\frac{1}{n} \log \nu_{1}, \quad R_{2}=\frac{1}{n} \log \nu_{2} .
$$

A rate-pair $\left(R_{1}, R_{2},\right)$ is said to be achievable if for every $\epsilon>0$ and sufficiently large $n$ there exists an $\left(n, 2^{n R_{1}}, 2^{n R_{2}}, \epsilon\right)$ code with strictly-causal SI for the channel $P_{Y \mid S, X_{1}, X_{2}}$. The capacity region of the channel with strictly-causal independent $\mathrm{SI}$ is the closure of the set of all achievable pairs $\left(R_{1}, R_{2}\right)$, and is denoted $\mathcal{C}_{\mathrm{s}-\mathrm{c}}^{\text {ind }}$. The superscript "ind" indicates that the two states are independent.

\section{The Single-State Scenario}

For the single-state scenario, an inner bound on $\mathcal{C}_{\mathrm{s}-\mathrm{c}}^{\mathrm{com}}$ was derived in [1] and later extended to many-transmitters in [3]. In the absence of cost constraints this bound can be described as follows: Let $\mathcal{P}_{\mathrm{s}-\mathrm{c}}^{\mathrm{com}}$ be the collection of all random variables $\left(U, V, X_{1}, X_{2}, W, Y\right)$ whose joint distribution satisfies

$$
P_{U, V, X_{1}, X_{2}, W, Y}=P_{W} P_{X_{1} \mid U} P_{X_{2} \mid U} P_{U} P_{V \mid W} P_{Y \mid W, X_{1}, X_{2}} .
$$

Note that (4) implies the Markov relations $X_{1} \multimap-U \multimap-X_{2}$ and $V \multimap-W \multimap-Y$, and that the triplet $\left(X_{1}, U, X_{2}\right)$ is independent of $(V, W)$. Let $\mathcal{R}_{\mathrm{s}-\mathrm{c}}^{\mathrm{com}}$ be the convex hull of the collection of all $\left(R_{1}, R_{2}\right)$ satisfying

$$
\begin{aligned}
R_{1} & \leq I\left(X_{1} ; Y \mid X_{2}, U, V\right) \\
R_{2} & \leq I\left(X_{2} ; Y \mid X_{1}, U, V\right) \\
R_{1}+R_{2} & \leq I\left(X_{1}, X_{2} ; Y \mid U, V\right) \\
R_{1}+R_{2} & \leq I\left(X_{1}, X_{2}, V ; Y\right)-I(V ; W)
\end{aligned}
$$

for some $\left(U, V, X_{1}, X_{2}, W, Y\right) \in \mathcal{P}_{\mathrm{s} \text {-c }}^{\text {com }}$.

Theorem 1 ([1]): $\mathcal{R}_{\mathrm{s}-\mathrm{c}}^{\mathrm{com}} \subseteq \mathcal{C}_{\mathrm{s}-\mathrm{c}}^{\mathrm{com}}$.

The achievability of this region is based on a Block-Markov scheme where at Block $\nu+1$ the transmitters send fresh private messages as well as a common message that is used to send a compressed version of the state sequence of Block $\nu$. The compression is of the Wyner-Ziv type with the side information being the channel outputs at Block $\nu$.

We next present a tighter inner bound. At Block $\nu+1$ we still use the MAC by sending private messages and a common message. The common message is still a compressed version of the state information from the previous block. The twist, however, is that the private messages need not be entirely composed of fresh information. The private message of Transmitter 1 has two parts. The first, of rate $R_{1}$, is indeed fresh information. But the second, of rate $R_{0}^{(1)}$, is a compressed version of the pair of sequences $\left(\mathbf{x}_{1}, \mathbf{w}\right)$ from Block $\nu$ (again with the side information being the received symbols in the previous block). Since Transmitter 1 knows which symbols it sent in the previous block, and since it knows the state of the channel in the previous block, it can compress the pair $\left(\mathbf{x}_{1}, \mathbf{w}\right)$. Likewise Transmitter 2. Using Gastpar's results on the compression of correlated sources with side information [4] we obtain the following bound: 
Theorem 2: The rate-pair $\left(R_{1}, R_{2}\right)$ is achievable if for some joint distribution of the form

$$
\begin{gathered}
P_{U, V, V_{1}, V_{2}, X_{1}, X_{2}, W, Y}= \\
P_{W} P_{X_{1} \mid U} P_{X_{2} \mid U} P_{U} P_{V \mid W} P_{V_{1} \mid W, X_{1}} P_{V_{2} \mid W, X_{2}} P_{Y \mid W, X_{1}, X_{2}}
\end{gathered}
$$

there exist nonnegative numbers $R_{0}^{(1)}$ and $R_{0}^{(2)}$ such that

$$
\begin{aligned}
R_{1}+R_{0}^{(1)} & \leq I\left(X_{1} ; Y, V_{1}, V_{2}, V \mid X_{2}, U\right)(7) \\
R_{2}+R_{0}^{(2)} & \leq I\left(X_{2} ; Y, V_{1}, V_{2}, V \mid X_{1}, U\right)(8) \\
R_{1}+R_{2}+R_{0}^{(1)}+R_{0}^{(2)} & \leq I\left(X_{1}, X_{2} ; Y, V_{1}, V_{2}, V \mid U\right)(9) \\
R_{0}+R_{1}+R_{2}+R_{0}^{(1)}+R_{0}^{(2)} & \leq I\left(X_{1}, X_{2} ; Y, V_{1}, V_{2}, V\right)
\end{aligned}
$$

and

$$
\begin{aligned}
R_{0}^{(1)} & \geq I\left(X_{1}, W ; V_{1} \mid V, V_{2}, Y\right) \\
R_{0}^{(2)} & \geq I\left(X_{2}, W ; V_{2} \mid V, V_{1}, Y\right) \\
R_{0} & \geq I\left(W ; V \mid V_{1}, V_{2}, Y\right) \\
R_{0}^{(1)}+R_{0}^{(2)} & \geq I\left(X_{1}, X_{2}, W ; V_{1}, V_{2} \mid V, Y\right) \\
R_{0}^{(1)}+R_{0} & \geq I\left(X_{1}, W ; V_{1}, V \mid V_{2}, Y\right) \\
R_{0}^{(2)}+R_{0} & \geq I\left(X_{2}, W ; V_{2}, V \mid V_{1}, Y\right) \\
R_{0}^{(1)}+R_{0}^{(2)}+R_{0} & \geq I\left(X_{1}, X_{2}, W ; V_{1}, V_{2}, V \mid Y\right) .
\end{aligned}
$$

If we only consider joint distributions where $V_{1}$ and $V_{2}$ are deterministic, and if we set $R_{0}^{(1)}, R_{0}^{(2)}$ to zero, we obtain the inner bound of [1]. Thus,

Remark 1: The proposed inner bound contains the inner bound of [1]

The following example shows that the inclusion can be strict.

Example 1: Consider a MAC with two binary inputs $\mathcal{X}_{1}=$ $\mathcal{X}_{2}=\{0,1\}$; a common state $W=\left(W_{0}, W_{1}\right) \in\{0,1\}^{2}$, where $W_{0}, W_{1}$ are IID with entropy

$$
H\left(W_{0}\right)=H\left(W_{1}\right)=1 / 2 ;
$$

and an output $Y=\left(Y_{1}, Y_{2}\right) \in\{0,1\}^{2}$ with

$$
\begin{aligned}
& Y_{1}=X_{1} \oplus W_{X_{2}} \\
& Y_{2}=X_{2} .
\end{aligned}
$$

Thus, if $X_{2}$ is equal to zero, then $Y_{1}$ is the mod-2 sum of $X_{1}$ and $W_{0}$, and otherwise it is the mod-2 sum of $X_{1}$ and $W_{1}$. We study the highest rate at which User 2 can communicate when User 1 transmits at rate 1 . We show that for this channel

$$
\max \left\{R_{2}:\left(1, R_{2}\right) \in \mathcal{R}_{\mathrm{s}-\mathrm{c}}^{\mathrm{com}}\right\}=0
$$

but

$$
\max \left\{R_{2}:\left(1, R_{2}\right) \in \mathcal{C}_{\mathrm{s}-\mathrm{c}}^{\mathrm{com}}\right\}=1 / 2,
$$

and that the rate-pair $(1,1 / 2)$ is in the new inner bound.

Proof: We first prove (15). To this end we note that if $\left(1, R_{2}\right)$ is achievable, then $R_{2}$ cannot exceed $1 / 2$. This can be shown using the full-cooperation outer-bound [1], which implies that $\left(R_{1}, R_{2}\right)$ can only be achievable if $R_{1}+R_{2} \leq 3 / 2$. Of more interest to us is the fact that the rate-pair $(1,1 / 2)$ is achievable. We demonstrate this using the new inner bound. Indeed, it is straightforward to verify that setting

$$
\begin{gathered}
R_{0}^{(1)}=R_{0}=0, \quad R_{0}^{(2)}=1 / 2, \\
V=V_{1}=0, \quad V_{2}=W_{X_{2}}, \\
U=0,
\end{gathered}
$$

$$
X_{1}, X_{2} \sim \text { IID Bernoulli } 1 / 2 \text {, }
$$

and

$$
\left(R_{1}, R_{2}\right)=(1,1 / 2)
$$

satisfies all the required inequalities. This choice corresponds to the following Block-Markov scheme: In the Block-Markov scheme Transmitter 1 sends its data uncoded. At Block $b+1$ Transmitter 2 sends $n$ bits, half of which are fresh data bits and half of which are used to describe the $n$-length sequence $\mathbf{w}_{\mathbf{x}_{2}}$ of the previous block. Note that Transmitter 2 does not describe the entire state sequence $\mathbf{w}$ of the previous block but only $\mathbf{w}_{\mathbf{x}_{2}}$. This latter sequence is known to Transmitter 2 at the beginning of Block $b+1$ thanks to the strictly-causal state information and because it knows the sequence $\mathbf{x}_{2}$ it transmitted in the previous block. And $n / 2$ bits suffice to describe this sequence because $W_{X_{2}}$ is of entropy $1 / 2$.

We now turn to proving (14). We fix some distribution $P_{U, V, X_{1}, X_{2}, W, Y}$ of the form (4), we assume that $\left(R_{1}=1, R_{2}\right)$ satisfy Inequalities (5), and we then prove that $R_{2}$ must be zero. Since $R_{1}=1$ and since $\mathcal{X}_{1}$ is binary, Inequality (5a) must hold with equality, and $X_{1}$ must be independent of $\left(X_{2}, U, V\right)$. By (4), this implies that

$$
X_{1} \text { is independent of }\left(X_{2}, U, V, W\right) .
$$

From (5a) (that we know holds with equality) and the fact that $R_{1}=1$ we also infer that

$$
\begin{aligned}
1 & =H\left(Y \mid X_{2}, U, V\right)-H\left(Y \mid X_{1}, X_{2}, U, V\right) \\
& =H\left(Y_{1} \mid X_{2}, U, V\right)-H\left(Y_{1} \mid X_{1}, X_{2}, U, V\right)
\end{aligned}
$$

where the second equality holds because $Y_{2}$ is a deterministic function of $X_{2}$. Since $Y_{1}$ is binary, $H\left(Y_{1} \mid X_{2}, U, V\right)$ is upperbounded by 1 , and we conclude from (17b) that

$$
\begin{aligned}
0 & =H\left(Y_{1} \mid X_{1}, X_{2}, U, V\right) \\
& =H\left(Y_{1} \oplus X_{1} \mid X_{1}, X_{2}, U, V\right) \\
& =H\left(W_{X_{2}} \mid X_{1}, X_{2}, U, V\right) \\
& =H\left(W_{X_{2}} \mid X_{2}, U, V\right)
\end{aligned}
$$

where the last equality follows from (17a).

We next show that

$$
U \multimap\left(X_{2}, V\right) \multimap W_{X_{2}} .
$$

To this end we note that, by (4), the pair $(V, W)$ is independent of $\left(U, X_{2}\right)$ and hence

$$
U \multimap-\left(X_{2}, V\right) \multimap-W .
$$

Since $W_{X_{2}}$ is a deterministic function of $\left(X_{2}, V, W\right)$, this implies (17d), because if $A \multimap-B \multimap-C$ forms a Markov chain 
then $A \multimap-B \multimap f(B, C)$. Having established (17d), we now obtain from (17c)

$$
H\left(W_{X_{2}} \mid X_{2}, V\right)=0 .
$$

We now focus on the case where $X_{2}$ is not deterministic

$$
\operatorname{Pr}\left[X_{2}=\eta\right]>0, \quad \eta \in\{0,1\},
$$

because if $X_{2}$ is deterministic then $R_{2}$ must be zero by (5b). We also assume that the PMF of $V$ is strictly positive

$$
\operatorname{Pr}[V=v]>0, \quad v \in \mathcal{V},
$$

because outcomes of the auxiliary random variable that have zero probability can be removed from $\mathcal{V}$ without affecting the inner bound. Since, by (4), $V$ is independent of $X_{2}$, it follows from $(17 \mathrm{~g})$ and $(17 \mathrm{~h})$ that

$$
\operatorname{Pr}\left[X_{2}=\eta, V=v\right]>0, \quad \eta \in\{0,1\}, v \in \mathcal{V} .
$$

This and (17f) imply that

$$
H\left(W_{\eta} \mid X_{2}=\eta, V=v\right)=0, \quad \eta \in\{0,1\}, v \in \mathcal{V} .
$$

Since, by (4), $X_{2}$ is independent of $(V, W)$ and, a fortiori, of $\left(V, W_{\eta}\right)$, it follows from $(17 \mathrm{j})$ that

$$
H\left(W_{\eta} \mid V=v\right)=0, \quad \eta \in\{0,1\}, v \in \mathcal{V} .
$$

Thus, $H\left(W_{\eta} \mid V\right)=0$, and since $W=\left(W_{0}, W_{1}\right)$,

$$
H(W \mid V)=0 .
$$

Consequently,

$$
\begin{aligned}
I(V ; W) & =H(W) \\
& =1,
\end{aligned}
$$

where the second equality follows from (12) and the independence of $W_{0}$ and $W_{1}$. From $(17 \mathrm{~m}),(5 \mathrm{~d})$, and the fact that $\mathcal{Y}$ has four elements we then conclude that $R_{1}+R_{2} \leq 1$. This combines with $R_{1}=1$ to establish that $R_{2}$ must be zero.

Terminating the Block-Markov scheme: To conclude the sketch of the achievability of the new inner bound, we still need to describe how the Block-Markov scheme is terminated. We thus assume that $B$ blocks have been transmitted, and we proceed to describe Blocks $B+1, B+2$, and $B+3$. We think about these blocks as "overhead," because they contain no fresh information. Fortunately, this overhead does not affect the throughput because we can choose $B$ very large.

The next lemma shows that if the full-cooperation capacity of the MAC without SI is zero, then the new inner bound contains only the rate-pair $(0,0)$ and is thus trivially an inner bound.

Lemma 1: If the capacity of the MAC without any side information but with full cooperation is zero, i.e., if

$$
\max _{P_{X_{1}, X_{2}}} I\left(X_{1}, X_{2} ; Y\right)=0
$$

then the proposed new inner bound contains only the all-zero rate tuple.
Proof: By (11g) and (10), we conclude that if $R_{1}, R_{2}$ is in the new inner bound, then for some joint distribution of the form (6)

$$
\begin{aligned}
R_{1}+ & R_{2} \\
\leq & I\left(X_{1}, X_{2} ; Y, V_{1}, V_{2}, V\right)-I\left(X_{1}, X_{2}, W ; V_{1}, V_{2}, V \mid Y\right) \\
= & I\left(X_{1}, X_{2} ; Y\right)+I\left(X_{1}, X_{2} ; V_{1}, V_{2}, V \mid Y\right) \\
& -I\left(X_{1}, X_{2}, W ; V_{1}, V_{2}, V \mid Y\right) .
\end{aligned}
$$

Consequently, if (18) holds and hence $I\left(X_{1}, X_{2} ; Y\right)$ is zero, then $R_{1}+R_{2}$ must be upper-bounded by $I\left(X_{1}, X_{2} ; V_{1}, V_{2}, V \mid Y\right)-I\left(X_{1}, X_{2}, W ; V_{1}, V_{2}, V \mid Y\right)$, which is nonpositive.

In view of Lemma 1 , it only remains to prove the achievability of the new inner bound when the full-cooperation capacity without SI is positive. The next lemma shows that we can also assume that the channel between Transmitter 1 (uninformed) and the receiver (informed) is of positive capacity and likewise from Transmitter 2 .

Lemma 2: If the channel between Transmitter 1 (uninformed) to the receiver (informed) is of zero capacity, i.e.,

$$
\max _{x_{2} \in \mathcal{X}_{2}} \max _{P_{X_{1}}} I\left(X_{1} ; Y, W \mid X_{2}=x_{2}\right)=0
$$

then the new inner bound contains only rate pairs $\left(R_{1}, R_{2}\right)$ with $R_{1}=0$ and $R_{2} \leq \max I\left(X_{2} ; Y\right)$. An analogous result holds if

$$
\max _{x_{1} \in \mathcal{X}_{1}} \max _{P_{X_{2}}} I\left(X_{2} ; Y, W \mid X_{1}=x_{1}\right)=0,
$$

Proof: We first prove that if a rate pair $\left(R_{1}, R_{2}\right)$ is in the new inner bound, and if (19) holds, then $R_{1}$ must be zero. Fix some joint distribution of the form (6) and let $\left(R_{1}, R_{2}\right)$ satisfy the inequalities of Theorem 2. We next argue that Hypothesis (19) implies

$$
I\left(X_{1} ; Y, V_{2}, V \mid X_{2}, U\right)=0 .
$$

Indeed,

$$
\begin{aligned}
I( & \left.X_{1} ; Y, V_{2}, V \mid X_{2}, U\right) \\
& \leq I\left(X_{1} ; Y, V_{2}, V \mid X_{2}, U, W\right) \\
& =I\left(X_{1} ; Y \mid X_{2}, U, W, V_{2}, V\right) \\
& =I\left(X_{1} ; Y \mid X_{2}, U, W\right), \\
& =I\left(X_{1} ; Y, W \mid X_{2}, U\right), \\
& \leq \max _{u \in \mathcal{U}} \max _{x_{2} \in \mathcal{X}_{2}} I\left(X_{1} ; Y, W \mid X_{2}=x_{2}, U=u\right) \\
& \leq \max _{u \in \mathcal{U}} \max _{x_{2} \in \mathcal{X}_{2}} \max _{X_{1} \mid U=u} I\left(X_{1} ; Y, W \mid X_{2}=x_{2}, U=u\right) \\
& =\max _{x_{2} \in \mathcal{X}_{2}} \max _{P_{X_{1}}} I\left(X_{1} ; Y, W \mid X_{2}=x_{2}\right)
\end{aligned}
$$

where the first line follows from

$$
X_{1} \multimap-\left(X_{2}, U\right) \multimap W
$$

the second from the chain rule and because

$$
X_{1} \multimap\left(X_{2}, U, W\right) \multimap\left(V_{2}, V\right)
$$


so $I\left(X_{1} ; V_{2}, V \mid X_{2}, U, W\right)$ is zero; the third from

$$
\left(X_{1}, Y\right) \multimap\left(X_{2}, U, W\right) \multimap\left(V_{2}, V\right) \text {; }
$$

the fourth again by (23); the fifth by upper bounding the average by the maximal value; the sixth by maximizing over the conditional distribution of $X_{1}$ given $U=u$; and the last because the maximization over $u$ on the RHS of (22f) is unnecessary.

Continuing our proof that $R_{1}$ must be zero, we note that (7) and (11a) imply

$$
\begin{aligned}
R_{1} \leq & I\left(X_{1} ; Y, V_{1}, V_{2}, V \mid X_{2}, U\right)-I\left(X_{1}, W ; V_{1} \mid V, V_{2}, Y\right) \\
= & I\left(X_{1} ; Y, V_{2}, V \mid X_{2}, U\right)+I\left(X_{1} ; V_{1} \mid X_{2}, U, Y, V_{2}, V\right) \\
& \quad-I\left(X_{1}, W ; V_{1} \mid V, V_{2}, Y\right) \\
= & I\left(X_{1} ; V_{1} \mid X_{2}, U, Y, V_{2}, V\right)-I\left(X_{1}, W ; V_{1} \mid V, V_{2}, Y\right) \\
= & H\left(V_{1} \mid X_{2}, U, Y, V_{2}, V\right)-H\left(V_{1} \mid X_{1}, X_{2}, U, Y, V_{2}, V\right) \\
& \quad+H\left(V_{1} \mid X_{1}, W, V, V_{2}, Y\right)-H\left(V_{1} \mid V, V_{2}, Y\right) \\
= & H\left(V_{1} \mid X_{2}, U, Y, V_{2}, V\right)-H\left(V_{1} \mid X_{1}, X_{2}, U, Y, V_{2}, V\right) \\
& \quad+H\left(V_{1} \mid X_{1}, W\right)-H\left(V_{1} \mid V, V_{2}, Y\right) \\
\leq & 0,
\end{aligned}
$$

where the second equality (third line) follows from (21), and where in the last inequality we have used

$$
H\left(V_{1} \mid X_{2}, U, Y, V_{2}, V\right) \leq H\left(V_{1} \mid V, V_{2}, Y\right)
$$

(conditioning reduces entropy) and

$$
H\left(V_{1} \mid X_{1}, X_{2}, U, Y, V_{2}, V\right) \geq H\left(V_{1} \mid X_{1}, W\right),
$$

which can be argued as follows:

$$
\begin{aligned}
H\left(V_{1} \mid X_{1}, X_{2}, U, Y, V_{2}, V\right) & \geq H\left(V_{1} \mid X_{1}, W, X_{2}, U, Y, V_{2}, V\right) \\
& =H\left(V_{1} \mid X_{1}, W\right),
\end{aligned}
$$

where the first inequality is because conditioning cannot increase entropy, and the second by (6), which implies that, conditional on $\left(X_{1}, W\right)$, the auxiliary random variable $V_{1}$ is independent of $\left(X_{2}, U, Y, V_{2}, V\right)$.

Having established that $R_{1}$ is zero, we now conclude from (11g) and (10)

$$
\begin{aligned}
R_{2}= & R_{1}+R_{2} \\
\leq & I\left(X_{1}, X_{2} ; Y, V_{1}, V_{2}, V\right)-I\left(X_{1}, X_{2}, W ; V_{1}, V_{2}, V \mid Y\right) \\
= & I\left(X_{1}, X_{2} ; Y\right)+ \\
& I\left(X_{1}, X_{2} ; V_{1}, V_{2}, V \mid Y\right)-I\left(X_{1}, X_{2}, W ; V_{1}, V_{2}, V \mid Y\right) \\
\leq & I\left(X_{1}, X_{2} ; Y\right) \\
= & I\left(X_{2} ; Y\right)+I\left(X_{1} ; Y \mid X_{2}\right) \\
= & I\left(X_{2} ; Y\right) .
\end{aligned}
$$

Lemma 2 shows that if either (19) or (20) holds, then the new inner bound is achievable. It thus only remains to prove its achievability when

$$
\max _{x_{2} \in \mathcal{X}_{2}} \max _{P_{X_{1}}} I\left(X_{1} ; Y, W \mid X_{2}=x_{2}\right)>0
$$

and

$$
\max _{x_{1} \in \mathcal{X}_{1}} \max _{P_{X_{2}}} I\left(X_{2} ; Y, W \mid X_{1}=x_{1}\right)>0,
$$

both of which we now assume.

We are now ready to describe the termination of the BlockMarkov scheme. Block $B+1$ is split into two parts. In the first, Transmitter 1 sends the $\mathbf{v}_{1}$-sequence of Block $B$ assuming that the receiver knows the state sequence $\mathbf{w}$ of Block $B+1$. This can be done (under this assumption) by (26). In the second, Transmitter 2 sends the $\mathbf{v}_{2}$-sequence of Block $B$ assuming that the receiver knows the state of Block $B+1$. This is possible by (27). In Block $B+2$ the transmitters cooperate to send the sequence $\mathbf{w}$ of Block $B+1$, and in Block $B+3$ they cooperate to send the $\mathbf{v}$ sequence of Block $B$.

Decoding is performed as follows. The decoder first decodes Block $B+3$ without any side-information and thus learns the sequence $\mathbf{v}$ of Block $B$. It then decodes Block $B+2$ (again without any side information) and learns the state sequence $\mathbf{w}$ of Block $B+1$. Now that it knows the state sequence of Block$B+1$, it can decode that block and learn the $\mathbf{v}_{1}$-sequence and the $\mathbf{v}_{2}$-sequence of Block $B$. From here on, it can proceed with the regular backward decoding: in decoding Block $b$ it knows the sequences $\mathbf{v}, \mathbf{v}_{1}$, and $\mathbf{v}_{2}$ of Block $b$ and it can therefore decode the common message and the messages transmitted by each of the transmitters in Block $b$. From this decoding it learns the private messages of Block $b$, and the sequences $\mathbf{v}$, $\mathbf{v}_{1}$, and $\mathbf{v}_{2}$ of Block $b-1$.

\section{The Double-State Scenario}

For the double-state scenario, an inner bound on $\mathcal{C}_{\mathrm{s}-\mathrm{c}}^{\text {ind }}$ was proposed in [2]. In the absence of cost constraints this bound can be described as follows: Let $\mathcal{P}_{\mathrm{s}-\mathrm{c}}^{\text {ind }}$ be the collection of all random variables $\left(V_{1}, V_{2}, S_{1}, S_{2}, X_{1}, X_{2}, Y\right)$ whose joint distribution satisfies

$$
\begin{aligned}
& P_{V_{1}, V_{2}, S_{1}, S_{2}, X_{1}, X_{2}, Y}= \\
& \quad P_{V_{1} \mid S_{1}} P_{V_{2} \mid S_{2}} P_{S_{1}} P_{S_{2}} P_{X_{1}} P_{X_{2}} P_{Y \mid S_{1}, S_{2}, X_{1}, X_{2}} .
\end{aligned}
$$

Note that (28) implies the Markov relations

$$
\begin{aligned}
& V_{1} \multimap-S_{1} \multimap-\left(V_{2}, Y, S_{2}\right) \\
& V_{2} \multimap-S_{2} \multimap-\left(V_{1}, Y, S_{1}\right) \\
& \left(V_{1}, V_{2}\right) \multimap\left(\left(S_{1}, S_{2}\right) \multimap-Y\right.
\end{aligned}
$$

and that $X_{1}, X_{2}$ are independent of each other and of the quadruple $\left(V_{1}, V_{2}, S_{1}, S_{2}\right)$. Let $\mathcal{R}_{\mathrm{s}-\mathrm{c}}^{\text {ind }}$ be the convex hull of the collection of all rate-pairs $\left(R_{1}, R_{2}\right)$ satisfying

$$
\begin{aligned}
0 & \leq R_{1} \leq I\left(X_{1} ; Y \mid X_{2}, V_{1}, V_{2}\right)-I\left(V_{1} ; S_{1} \mid Y, V_{2}\right) \\
0 & \leq R_{2} \leq I\left(X_{2} ; Y \mid X_{1}, V_{1}, V_{2}\right)-I\left(V_{2} ; S_{2} \mid Y, V_{1}\right) \\
R_{1}+R_{2} & \leq I\left(X_{1}, X_{2} ; Y \mid V_{1}, V_{2}\right)-I\left(V_{1}, V_{2} ; S_{1}, S_{2} \mid Y\right)
\end{aligned}
$$

for some $\left(V_{1}, V_{2}, S_{1}, S_{2}, X_{1}, X_{2}, Y\right) \in \mathcal{P}_{\text {s-c }}^{\text {ind }}$.

Theorem 3 ([2]): $\mathcal{R}_{\mathrm{s}-\mathrm{c}}^{\text {ind }} \subseteq \mathcal{C}_{\mathrm{s}-\mathrm{c}}^{\text {ind }}$.

The proof is based on a scheme where lossy versions of the state sequences are conveyed to the decoder using distributed Wyner-Ziv compression [4] and Block-Markov encoding for 
the MAC, to transmit the messages and the Wyner-Ziv codewords. The channel output serves as the decoder's SI in the distributed Wyner-Ziv code. Since the two components of the source are independent, there is no direct cooperation between the encoders via a common message as in single-state scenario. Instead, each user spends part of its private rate on the transmission of its Wyner-Ziv codeword.

An improved inner bound was proposed by Li et al. in [3]. There it was shown that the improved inner bound always contains the inner bound of [2], and it was conjectured that there are cases where the inclusion is strict. We next present the inner bound of Li et al. and then show that the inclusion can, indeed, be strict.

Li et al. consider all joint distributions of the form

$$
\begin{aligned}
& P_{V_{1}, V_{2}, S_{1}, S_{2}, X_{1}, X_{2}, Y}= \\
& \quad P_{V_{1} \mid S_{1}, X_{1}} P_{V_{2} \mid S_{2}, X_{2}} P_{S_{1}} P_{S_{2}} P_{X_{1}} P_{X_{2}} P_{Y \mid S_{1}, S_{2}, X_{1}, X_{2}}
\end{aligned}
$$

and prove the achievability of rate pairs $\left(R_{1}, R_{2}\right)$ satisfying

$$
\begin{aligned}
R_{1} \leq I\left(X_{1}, V_{1} ; Y \mid X_{2}, V_{2}\right)-I\left(V_{1} ; S_{1} \mid X_{1}\right) \\
R_{2} \leq I\left(X_{2}, V_{2} ; Y \mid X_{1}, V_{1}\right)-I\left(V_{2} ; S_{2} \mid X_{2}\right) \\
R_{1}+R_{2} \leq I\left(X_{1}, X_{2}, V_{1}, V_{2} ; Y\right) \\
\quad-I\left(V_{1} ; S_{1} \mid X_{1}\right)-I\left(V_{2} ; S_{2} \mid X_{2}\right) .
\end{aligned}
$$

Roughly speaking, the improvement in the inner bound is the result of Transmitter 1 compressing the pair $\left(\mathbf{s}_{1}, \mathbf{x}_{1}\right)$ from the previous block (with the outputs from the previous block serving as side information) and not just $\mathbf{s}_{1}$ and likewise for Transmitter 2. We next show, by example, that the bound of Li et al. can, indeed, be tighter than that of Theorem 3

The example is very similar to Example 1. In fact, the channel is as in Example 1, but with the state $S_{1}$ being null (deterministic) and the state $S_{2}$ consisting of the pair $\left(W_{0}, W_{1}\right)$ of Example 1:

$$
S_{1}=0 \quad S_{2}=\left(W_{0}, W_{1}\right),
$$

where $W_{0}, W_{1}$ are IID binary random variables, each of entropy $1 / 2$.

The rate pair $\left(R_{1}, R_{2}\right)=(1,1 / 2)$ is in the inner bound of Li et al.. To see this we set $V_{1}=0$ and $V_{2}=W_{X_{2}}$ with $X_{1}, X_{2}$ IID random bits. However, as we next prove, the pair $(1,1 / 2)$ is not in $\mathcal{R}_{\mathrm{s}-\mathrm{c}}^{\text {ind }}$.

We prove this by showing that if $\left(1, R_{2}\right)$ is in $\mathcal{R}_{\mathrm{s}-\mathrm{c}}^{\text {ind }}$, then $R_{2}$ must be zero. Suppose then that $\left(1, R_{2}\right) \in \mathcal{R}_{\mathrm{s}-\mathrm{c}}^{\text {ind }}$. Since $S_{1}$ is null, it follows from the structure (28) of the joint distribution, that $V_{1}$ must be independent of all the other random variables. Consequently, we can strike it out from (30), (31), and (32). Since $R_{1}=1$, it follows from (30) that $X_{1}$ must be Bernoulli(1/2) and that $H\left(X_{1} \mid X_{2}, V_{2}, Y\right)$ must be zero. This implies that $H\left(W_{X_{2}} \mid X_{2}, V_{2}, Y\right)$ must also be zero (because $\left.X_{1}=Y_{1} \oplus W_{X_{2}}\right)$. Consequently, $H\left(W_{X_{2}} \mid X_{2}, V_{2}, Y_{1}\right)$ must also be zero (because $Y_{2}=X_{2}$ ). This implies that

$$
H\left(W_{X_{2}} \mid X_{2}, V_{2}\right)=0
$$

because $X_{1}$ is Bernoulli(1/2) and independent of $\left(X_{2}, V_{2}, W\right)$, so $Y_{1}$, which is equal to $X_{1} \oplus W_{X_{2}}$, must also be independent of $\left(X_{2}, V_{2}, W\right)$. Equation (35b) is reminiscent of (17f) (with $V_{2}$ replacing $V$ ).

As in Example 1, we now distinguish between two cases depending on whether $X_{2}$ is deterministic or not. If it is deterministic, then the rate $R_{2}$ must be zero by (31). Consider now the case when it is not. In this case $\operatorname{Pr}\left[X_{2}=\eta\right]$ is positive for all $\eta \in\{0,1\}$. Since $V_{2}$ is independent of $X_{2}$ (by (28)), and since without changing the inner bound we can assume that $\operatorname{Pr}\left[V_{2}=v_{2}\right]$ is positive for all $v_{2} \in \mathcal{V}_{2}$, it follows that in this case

$$
\operatorname{Pr}\left[X_{2}=\eta, V_{2}=v_{2}\right]>0, \quad \eta \in\{0,1\}, v_{2} \in \mathcal{V}_{2} .
$$

This combines with (35b) to imply that

$$
H\left(W_{\eta} \mid X_{2}=\eta, V_{2}=v_{2}\right)=0, \quad \eta \in\{0,1\}, v_{2} \in \mathcal{V}_{2} .
$$

This implies that

$$
H\left(W_{\eta} \mid V_{2}=v_{2}\right)=0, \quad \eta \in\{0,1\}, v_{2} \in \mathcal{V}_{2},
$$

because, by (28), $X_{2}$ is independent of $\left(V_{2}, S_{2}\right)$ and hence $a$ fortiori of $\left(V_{2}, W_{\eta}\right)$. Thus, $H\left(W_{\eta} \mid V_{2}\right)=0$, and since $S_{2}=$ $\left(W_{0}, W_{1}\right)$,

$$
H\left(S_{2} \mid V_{2}\right)=0
$$

Consequently,

$$
I\left(V_{2} ; S_{2}\right)=H\left(S_{2}\right)=1 .
$$

This implies that also

$$
I\left(V_{2} ; S_{2} \mid Y\right)=1
$$

because $Y$ is independent of $\left(V_{2}, S_{2}\right)$. It now follows from (35h), the fact that $V_{1}$ is deterministic, and from (31) that $R_{2}$ must be zero.

\section{SUMMARY}

We have presented an improved inner bound on the capacity region of the memoryless multiple-access channel that is controlled by an IID state that is known strictly causally to the two encoders. This bound contains the bound of [1], and we have provided an example showing that the inclusion can be strict.

We also adapted this example to a memoryless multipleaccess channel that is governed by two independent states, where each transmitter knows one of the states strictly causally. The resulting example demonstrates that-as conjecture by $\mathrm{Li}$ et al. [3] - the inner bound of Li et al. can be strictly tighter than that of [2].

\section{REFERENCES}

[1] A. Lapidoth and Y. Steinberg, "The multiple-access channel with causal and strictly causal side information at the encoders," in Proceedings International Zurich Seminar, March 2010.

[2] A. Lapidoth and Y. Steinberg, "The multiple access channel with two independent states each known causally to one encoder,' in Proceedings IEEE International Symposium on Information Theory (ISIT), June 2010.

[3] M. Li, O. Simeone, and A. Yener, "Multiple access channels with states causally known at transmitters," Preprint. arXiv:1011.6639v1 30 November 2010.

[4] M. Gastpar, "On Wyner-Ziv networks," in Proc. 37th Asilomar Conf. Signals, Systems, and Computers, Asilomar, CA, Nov. 2003. 